\title{
Grain Orientation and Strain Measurements in Sub-Micron wide Passivated Individual Aluminum Test Structures
}

N. Tamura ${ }^{1}$, B. C. Valek ${ }^{2}$, R. Spolenak ${ }^{3}$, A. A. MacDowell ${ }^{1}$, R. S. Celestre ${ }^{1}$, H.A.Padmore ${ }^{1}$, W. L. Brown ${ }^{3}$, T. Marieb ${ }^{5}$, J. C. Bravman' ${ }^{2}$ B. W. Batterman ${ }^{1}$ and J. R. Patel ${ }^{1,4}$

${ }^{1}$ ALS/LBNL, 1 Cyclotron Road, Berkeley CA 94720, USA

${ }^{2}$ Dept. of Mat. Sci. \& Eng., Stanford University, Stanford, CA 94305, USA

${ }^{3}$ Bell Laboratories, Lucent Technologies, Murray Hill NJ 07974, USA

${ }^{4}$ SSRL/SLAC, Stanford University, Stanford, CA 94309, USA

${ }^{5}$ Intel Corp., Portland, OR 97124, USA

\begin{abstract}
An X-ray microdiffraction dedicated beamline, combining white and monochromatic beam capabilities, has been built at the Advanced Light Source. The purpose of this beamline is to address the myriad of problems in Materials Science and Physics that require submicron x-ray beams for structural characterization. Many such problems are found in the general area of thin films and nano-materials. For instance, the ability to characterize the orientation and strain state in individual grains of thin films allows us to measure structural changes at a very local level. These microstructural changes are influenced heavily by such parameters as deposition conditions and subsequent treatment. The accurate measurement of strain gradients at the micron and sub-micron level finds many applications ranging from the strain state under nano-indenters to gradients at crack tips. Undoubtedly many other applications will unfold in the future as we gain experience with the capabilities and limitations of this instrument. We have applied this technique to measure grain orientation and residual stress in single grains of pure Al interconnect lines and preliminary results on post-electromigration test experiments are presented. It is shown that measurements with this instrument can be used to resolve the complete stress tensor (6 components) in a submicron volume inside a single grain of $\mathrm{Al}$ under a passivation layer with an overall precision of about $20 \mathrm{MPa}$. The microstructure of passivated lines appears to be complex, with grains divided into identifiable subgrains and noticeable local variations of both tensile/compressive and shear stresses within single grains.
\end{abstract}

\section{INTRODUCTION}

The reliability of integrated circuits depends on the time to failure of the polycrystalline $\mathrm{Al}$ or $\mathrm{Cu}$ based lines used as device interconnections. Understanding interconnect failure mechanisms in order to predict and improve their lifetime is therefore a major concern in the semiconductor industry. Electromigration is the material transport resulting from the momentum transfer of the electron wind on the constitutive atoms under high current density. Damage by void formation, growth, and migration, ultimately causing open circuit failure, was very early identified as the main mechanism responsible for failure [1]. Voids form to overcome high stresses, which develop in the material during both fabrication and electromigration. The elevated residual stress, which builds up in the lines during the fabrication process, is essentially due to the thermal mismatch between the line, substrate, and surrounding oxide (passivation layer). Electromigration leads to the development of a compressive stress at the anode with 
possible hillock formation and of a tensile stress at the cathode with void formation. In the ideal case, the resulting stress gradient would counterbalance the atomic flux due to electromigration, leading to a steady state regime with no further damage ("immortal line"). Accurate measurements of this stress gradient as a function of such parameters as microstructure, initial residual stress state, line aspect ratio, chemical composition, and nature of passivation layer, constitute important inputs for electromigration damage modeling. On the other hand, void dynamics have been shown to be rather complex, with behavior such as drifting and coalescence observed [2,3]. Voids preferentially appear at grain boundaries or other imperfections on the lines and their locations depend on the microstructure and on the local stress state of the individual grains. Though progress has been recently made on the simulation of void dynamics [4-6], a full understanding of void formation is limited due to the lack of experimental information on microstructure and stress at the submicron level.

\section{EXPERIMENTAL PROCEDURE}

The beamline has a unity magnification toroidal mirror that produces a 50 by 200 micron focus just inside an x-ray hutch at the position of an x-y slit. The beam path in the hutch consists of source defining slits, a four bounce Ge or Si monochromator, followed by elliptically bent Kirkpatrick-Baez mirror pair which focuses the beam from the slits to sub micron dimensions $(0.8 \times 0.8$ microns FWHM $)$. An important feature of this arrangement is the ability to switch between white and monochromatic beams that are essential for characterizing crystal grains in the sub-micron range. The sample stage rests on a state of the art six-circle diffractometer equipped with encoders in the main rotation stages calibrated to a second of arc. The detector is a $4 \mathrm{~K} \times 4 \mathrm{~K}$ CCD (Bruker) with a 9x9 $\mathrm{cm}$ view area mounted on a detector arm that can be positioned around the sample. The detector itself can also be positioned within 1 micron over 40 centimeters along the detector arm.

Diffraction experiments at the sub-micron level raise certain issues not encountered or ignored in conventional diffraction. One overriding concern is, except for translations within the focal plane under the small beam, the sample has to remain fixed (i.e. no rotation can be allowed). Since the sphere of confusion of modern, well-constructed diffractometers is more than 10 microns, any rotation would move the sub-micron region of interest out of the beam. We have facilities for precision translation of the specimen to allow us to scan different crystal regions or grains. The equipment described above enables us to keep the sample fixed and rotate the CCD to the desired position around the sample. A similar technique is used at the APS [7-9] and we apply it to both $\mathrm{Al}$ interconnects (this paper) and damascene $\mathrm{Cu}$ interconnects [10].

The samples investigated are pure Al two level test structures 10 or $30 \mu \mathrm{m}$ long, $0.7 \mu \mathrm{m}$ wide and $0.75 \mu \mathrm{m}$ thick. Shunt layers of Ti cover the bottom and the top of the lines. The lines are passivated under $0.7 \mu \mathrm{m}$ of oxide (PETEOS). The test lines are connected to the Al pads by $\mathrm{W}$ vias.

The samples were scanned with white beam using a $0.5 \mu \mathrm{m}$ interval between images to obtain a grain orientation map of the entire structure. Next both the white and monochromatic beam features of this instrument were used to determine the stress state in individual grains under electromigration conditions at $225^{\circ} \mathrm{C}$. 


\section{RESULTS AND DISCUSSION}

The texture scan on the 10x0.7x0.75 microns passivated $\mathrm{Al}$ interconnect reveals a peculiar microstructure with overlapping grains of dimensions ranging from less than a micron to 3-4 microns in length. All of the 66 Laue patterns display at least two Al grains. The texture map itself does not immediately reveal the actual grain size because of the inability of the analysis software to adequately discriminate between top and bottom overlapping grains (the depth sensitivity of the technique is too weak to resolve the stacking sequence in a 0.75 thick sample), or between two neighboring grains giving similar intensity ratios. On the other hand, it is able to effectively find and index several grains in composite Laue patterns, so that the actual position and size of each single grain can be inferred. The analysis of this particular line reveals 4 different grains over 3 microns in size and several other minor micron size grains (the total number of detected grains is 17). Strictly speaking, the line microstructure is not of bamboo type. Some of the grains clearly overlapped and this seems to be a feature of this particular line since the investigation of other lines does not show systematic composite Laue patterns. This test structure does demonstrate the ability of the technique to probe buried grains. It also shows that even if a line seems to be bamboo-like when surface features are examined, it may contain boundaries which are far from being perpendicular to the surface and could constitute fast diffusion paths during electromigration. The analysis of the orientation of these grains reveals a rather poor fiber texture with out-of-plane (111) reflections ranging from 2 to $10^{\circ}$ from the surface normal.

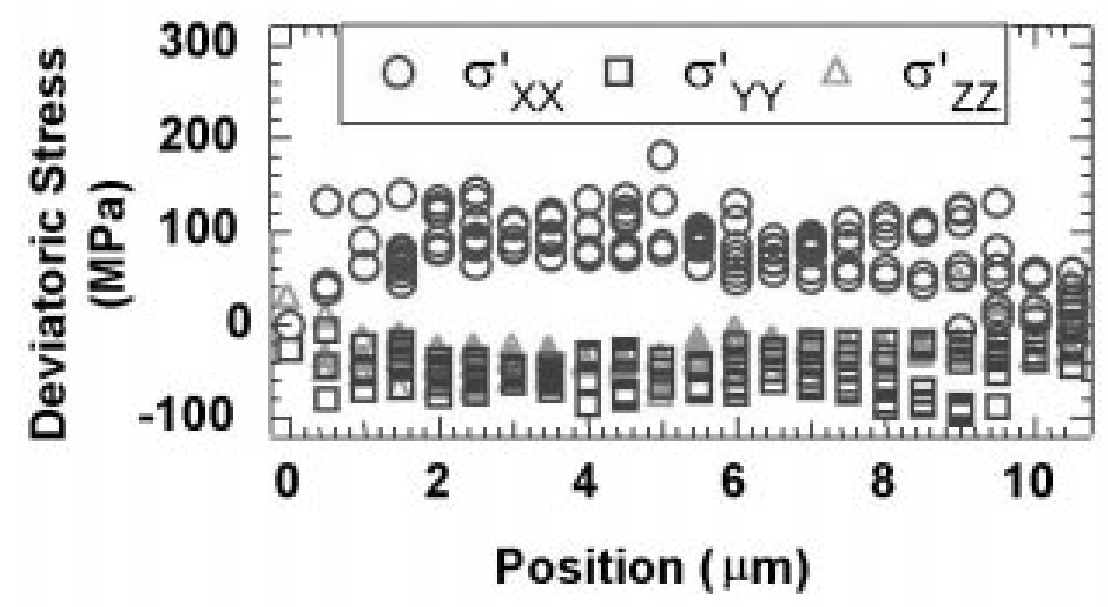

Fig 1.- Cumulative data on the deviatoric residual stress in the $10 \mu \mathrm{m}$ long wire
The deviatoric residual stress tensor [11] has been computed for each grain on each image and the results are cumulated in Fig 1. The deviatoric stress tensor is calculated from the deviatoric strain tensor by using the anisotropic stiffness constants of the material recalculated in the sample reference frame (we follow the usual convention labeling the $\mathrm{X}, \mathrm{Y}$ and $\mathrm{Z}$ directions along the line, across the line, and along the surface normal respectively).

The plot shows a decrease of the stress values at the two ends of the lines. This indicates that grains from the pads, expected to be only slightly strained, are also hit by the microbeam in these areas. In the wire, the grains are tensile along the direction of the wire (X) and compressed along the two other directions ( $\mathrm{Y}$ and $\mathrm{Z}$ ) by approximately the same amount [the terms tensile and compressive refers to the deviatoric component of the stress only and not the complete stress]. 
This result is in agreement with the observation of Hosoda et al. [12] who studied the stress values as a function of the aspect ratio (width/thickness) of the line. According to their work, an aspect ratio of one gives equal $\mathrm{Y}$ and $\mathrm{Z}$ stress components. The absolute differences between the $\mathrm{X}$ component and the Y,Z components is about 120-130 $\mathrm{MPa}$. These values are comparable to those obtained by other authors [13] even if they cannot be directly compared because of differences in aspect ratio, fabrication process, and passivation layers.
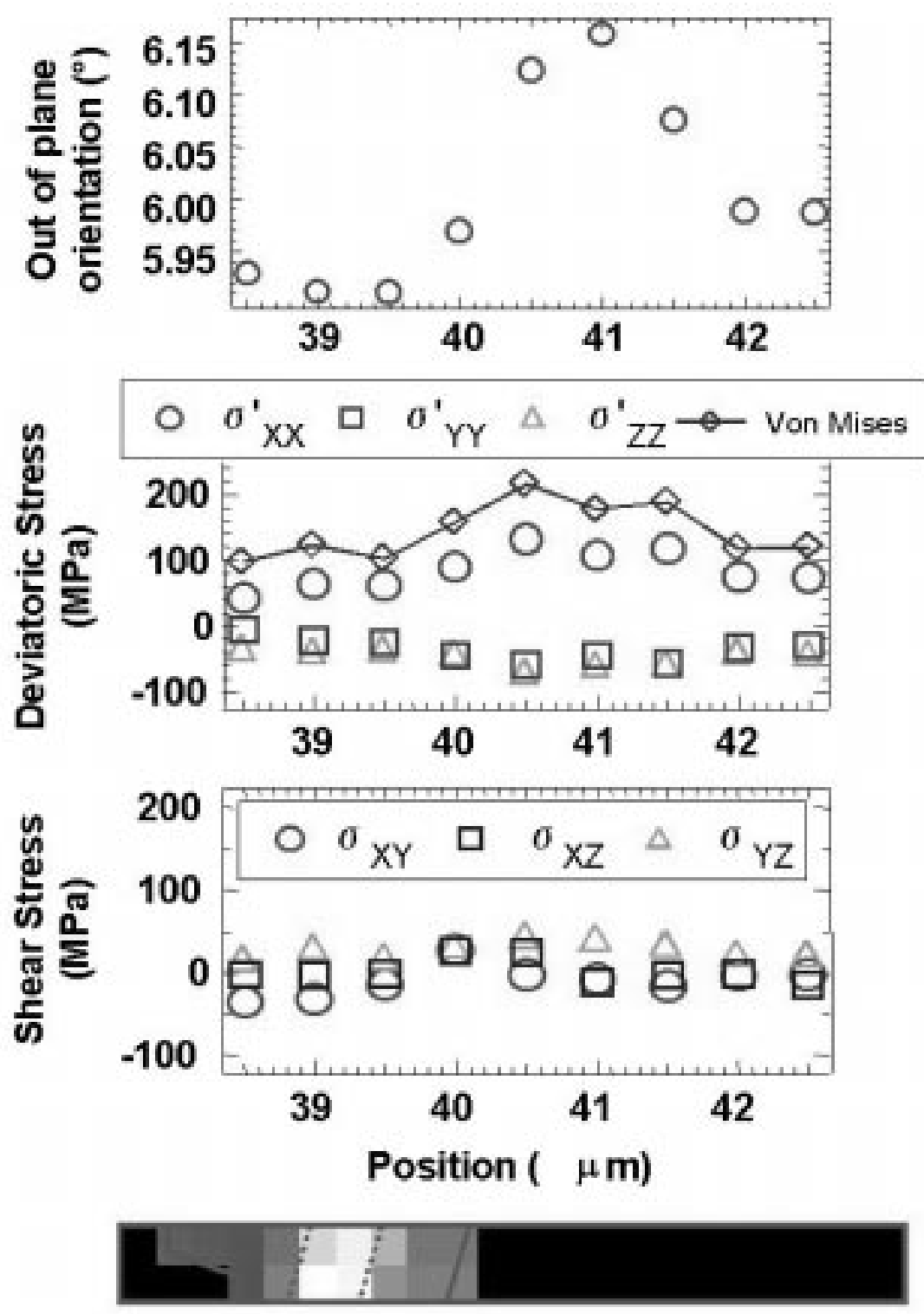

Fig. 2.- Texture and deviatoric stresses in a single grain of a pure Al interconnect. The bottom picture is a texture map of the grain. Lines have been added as guidelines in delimiting subgrains. The top plot shows orientation variations (tilts of the out-of-plane (111) with respect to the surface normal). The middle plots represent deviatoric stresses along $X, Y, Z$. and variations of the shear components of the stress.
A study of texture and strain/stress variations within individual grains is rich in detail (Fig. 2). The largest grains are made of subgrains about a micron in size stacked mainly in the direction of the line, as revealed by the shifts of the Laue spots as we scan along the grain. Since the indexation of the Laue patterns provides the full orientation matrices of each subgrain, the tilt axis together with the corresponding tilt angles between the subgrains can be computed. Whereas different neighboring grains are tilted with respect to each other mainly around the normal (111) direction, the tilt axis directions between subgrains are so far randomly distributed. The misorientation between subgrains is about half a degree. Each subgrain in turn reveals slight orientation contrast indicating that each of them is subdivided into finer submicron subgrains with smaller misorientations, giving to the whole microstructure a mosaic aspect.

The deviatoric stress profiles of individual grains show slight variation along them (Fig 2.). Larger bumps, which sometimes occurred at the boundaries between two subgrains, could be 
artifacts due to the fitting process of overlapping peaks coming from these two subgrains. The Von Mises stress, which is a measure of the distortion of the material, is often used as a reliability criterion in the industry. If the Von Mises stress locally exceeds the yield stress of the material, plastic deformation will occur. The observed tensile stress along the direction of the line and the observed subgrain microstructure are clear indications that plastic deformation occurred during or after the fabrication process of the line to partially relieve the resulting high stresses.

\begin{tabular}{|c|c|c|c|c|c|}
\hline Stress in & Initial & Final & $150^{\circ} \mathrm{C}$ & $75^{\circ} \mathrm{C}$ & $25^{\circ} \mathrm{C}$ \\
\hline $\mathrm{MPa}$ & $225^{\circ} \mathrm{C}$ & $225^{\circ} \mathrm{C}$ & & & \\
\hline$\sigma_{X X}^{\prime}$ & 37.3 & -2.8 & 39.9 & 81.2 & 118.4 \\
\hline$\sigma_{Y Y}^{\prime}$ & -10.3 & 28 & 22.2 & -6.1 & -20 \\
\hline$\sigma_{\mathrm{ZZ}}^{\prime}$ & -26.9 & -25.2 & -61.9 & -74.9 & -97.8 \\
\hline
\end{tabular}

Table 1.- Evolution of the deviatoric stress in a single grain before and after an electromigration test at $225^{\circ} \mathrm{C}$ and during cooling to room temperature

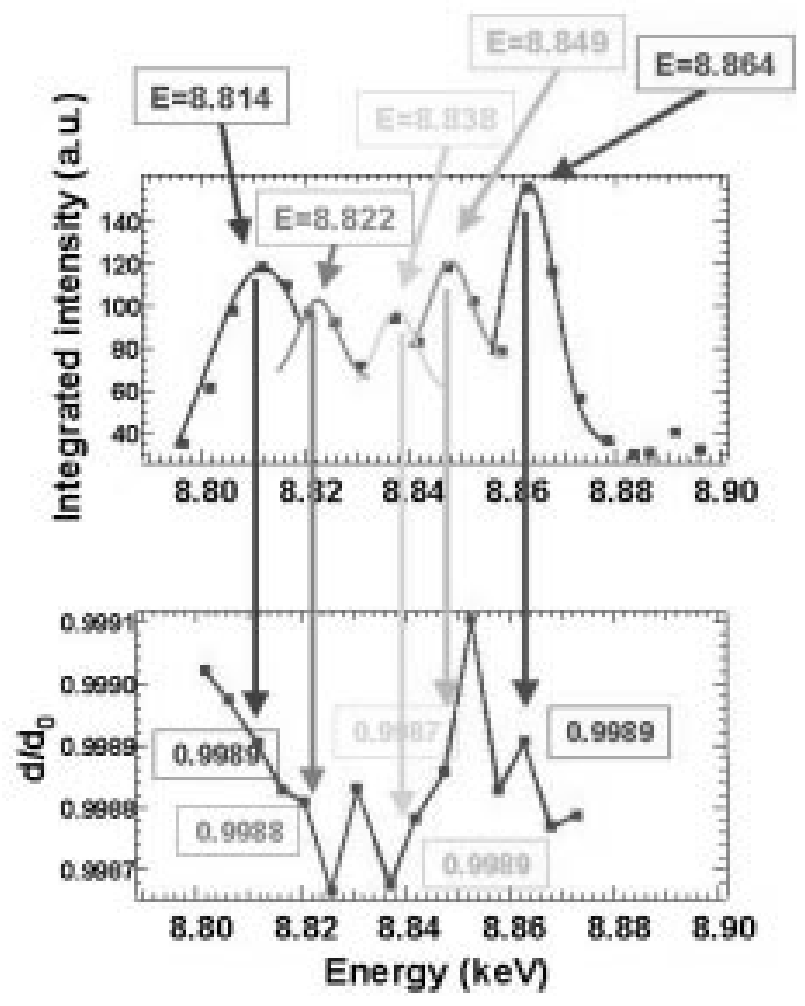

Fig. 3.- Energy scan of the (4 2 2) reflection at $225^{\circ} \mathrm{C}$
The deviatoric stress changes in the 30x $0.7 \times 0.75 \mu \mathrm{m}$ line after electromigration failure and during cooling is shown in Table 1 for a grain near the anode end. The results indicate a clear change in the deviatoric stress during electromigration. The $\mathrm{Z}$ component didn't change but the deviatoric stress along the $\mathrm{X}$ direction becomes compressive. The total change in stress (hydrostatic + deviatoric) before and after failure is $-76 \mathrm{MPa},+4 \mathrm{MPa}$ and $-25 \mathrm{MPa}$ along the $\mathrm{X}, \mathrm{Y}, \mathrm{Z}$ directions respectively. When cooling down the deviatoric component of the stress in the grain becomes tensile along the $\mathrm{X}$ direction and compressive along the $\mathrm{Z}$ direction. The total change in stress (hydrostatic + deviatoric) between $225^{\circ} \mathrm{C}$ and $25^{\circ} \mathrm{C}$ is $279.5 \mathrm{MPa}$, 110.3 $\mathrm{MPa}$ and $86 \mathrm{MPa}$ along the $\mathrm{X}, \mathrm{Y}$ and $\mathrm{Z}$ directions respectively.

The Laue patterns give the angular positions of the diffracted beams and their approximate energy can be deduced (assuming zero hydrostatic strain).

The small energy range around the peak can therefore be scanned with the monochromator and the peak position can be recorded with the CCD detector as a function of the incoming beam energy. Fig. 3 shows an example of such an energy scan. It was taken with a broad monochromatic beam of 3 by $3 \mu \mathrm{m}$ in order to illuminate the whole grain and see its 
microstructure. The energy peak is very irregular in shape with subpeaks corresponding to individual subgrains. Each subpeak can be fit to a gaussian function and the absolute value of the strain/stress is calculated for each of the subgrains. The observation indicates only a slight variation in strain. On the other hand, each subpeak has a certain width $(5-15 \mathrm{eV}$ wide) compared to an energy peak of a perfect Si sample (3-4 eV). These subpeak widths reflect the texture variation within the subgrains, confirming the mosaic nature of the microstructure in passivated $\mathrm{Al}$ interconnects.

\section{CONCLUSION}

We have demonstrated a new experimental $\mathrm{X}$-ray microdiffraction technique, which is able to resolve the complete strain and stress tensors at submicron level in buried grains. The technique does not necessitate any sample preparation so that the true stress and texture state are measured. It was shown that the microstructure of thin Al lines under passivation is extremely complex and rich with both stress and texture variations within single grains. The largest grains have a mosaic substructure resulting from plastic deformation during fabrication.

\section{ACKNOWLEDGEMENT}

The Advanced Light Source is supported by the Director, Office of Science, Office of Basic Energy Sciences, Materials Sciences Division, of the U.S. Department of Energy under Contract No. DE-AC03-76SF00098 at Lawrence Berkeley National Laboratory.

\section{REFERENCES}

[1] I.A. Blech and H. Sello, Physics of failure in Electronics Series proceedings (USAF Rome Air Development center Reliability, Rome, NY, 1967), Vol. 5, p. 496

[2] E. Arzt, O. Kraft, W.D. Nix, and J.E. Sanchez, Jr., J. Appl. Phys., 76, 1563 (1994)

[3] T. Marieb, P. Flinn, J. C. Bravman, D. Gardner, and M. Madden, J. Appl. Physics, 78, 10261032 (1995)

[4] M. R. Gungor and D. Maroudas, J. Appl. Phys., 85, 2233-2246 (1999)

[5] Y.-J. Park, V. K. Andleigh, and C. V. Thompson, J. Appl. Phys., 85, 3546-3555 (1999)

[6] R.-J. Gleixner and W. D. Nix, J. Appl. Phys., 86, 1932-1944

[7] J.-S. Chung, and G. E. Ice, J. Appl. Physics, 86, 5249-5255 (1999)

[8] J.-S. Chung, N. Tamura, G. E. Ice, B. C. Larson, J. D. Budai, W. Lowe, Mat. Res. Soc. Symp. Proc, 563, 169-174 (1999)

[9] N. Tamura, J.-S. Chung, G.E. Ice, B. C. Larson, J. D. Budai, J. Z. Tischler, M. Yoon, E. L. Williams, and W. P. Lowe, Mat. Res. Soc. Symp. Proc, 563, 175-180 (1999)

[10] R. Spolenak, D.L. Barr, M.E. Gross, K. Evans-Lutherodt, W.L. Brown, N. Tamura, A.A. MacDowell, R.S. Celestre, H.A.Padmore, J.R. Patel, B.C. Valek, J.C. Bravman, P. Flinn, T. Marieb, R.R. Keller, B.W. Batterman, Mat. Res. Soc. Symp. Proc., submitted (2000)

[11] I. C. Noyan and J. B. Cohen, Residual Stress: Measurement by Diffraction and Interpretation (Springer, New York, 1987), p. 33

[12] T. Hosoda, H. Yagi, and H. Tsuchikawa, 1989 International reliability Physics Symposium Proceedings, IEEE, p. 202-206 (1989)

[13] P. R. Besser, $X$-ray determination of thermal strains and stresses in thin aluminum films and lines, PhD, Stanford University (1993) 